\title{
Merits of a harmonised system to classify drug-related problems in Singapore
}

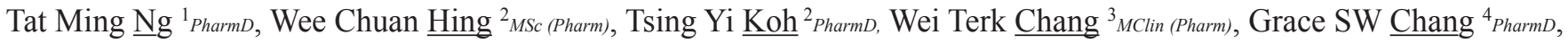
Jian Wei Heng ${ }^{4}$ PharmD, Isnarti Bte Abuaman ${ }^{4}$, Beng Yi Sia ${ }^{1}$ MSc (Pharm), Yik Chuen $\underline{\text { Saw }}{ }^{3}{ }_{\text {MClin (Pharm), Daphne }} \underline{\text { Chan }}^{5}{ }_{B \text { Pharm, }}$ Chwee Huat Tan ${ }^{5}$ MSc (Pharm), Wei Shan Fan ${ }^{6}{ }_{\text {MClin (Pharm), Franky Franky }}^{6}{ }_{B C G P}$, Poh Ching Tan $^{7}{ }_{\text {MClin (Pharm), }}$ Cheryl WY Tan ${ }^{7}$ PharmD, Joanne HL $\underline{\text { Sng }}{ }^{8}$ PharmD, Chun Wei Yap ${ }^{9}{ }_{\text {PhD }}$, Shanti Uma Devi d/o Gnanamani ${ }^{10}{ }_{\text {Bsc (Pharm), }}$ Doreen SY Tan ${ }^{4,11}$ PharmD

A drug-related problem (DRP) is commonly defined as an event or circumstance involving drug treatment that actually or potentially interferes with the optimal outcome of a patient's medical care. It broadly includes events related to errors, adverse effects or adherence issues. ${ }^{1}$ DRPs are associated with increased healthcare costs and hospital admissions, prolonged hospital stays, reduced quality of life and increased mortality. ${ }^{2,3}$ Patients with multiple comorbidities and polypharmacy are at risk of DRPs, present during or immediately after discharge. ${ }^{4}$ Polypharmacy and DRPs are also associated with readmissions in Singapore. ${ }^{5}$

Healthcare professionals and primarily pharmacists help to identify and resolve DRPs daily in their course of work in different settings such as hospitals, nursing homes, polyclinics, community pharmacies and other community-based care facilities. ${ }^{6-10}$ In many pharmacy departments, data on DRPs and their resolution have also been collected as part of medication safety surveillance as well as to facilitate quality and safety improvements and education initiatives. In addition, such data may also be used to estimate pharmacy workload as DRPs can lead to reworking of prescriptions. A recently conducted cross-sectional study of DRPs detected $38.3 \%$ of 379 hospitalisations with one or more DRPs. Over $90 \%$ of these DRPs were preventable, with an estimated median admission cost of SGD1,424 and interquartile range of SGD1,068-2,678. ${ }^{11}$ The detection and resolution of DRPs optimises medication use, maximises the utility of medication costs and potentially reduces healthcare costs in Singapore's healthcare system. ${ }^{12}$
Currently, there is no harmonised classification system across Singapore institutions. Different methods of classifications result in difficulties interpreting reports of DRP prevalence rates and causes. ${ }^{13}$ To tackle these DRPs as a healthcare system, it is important that institutions share similar classifications. This will enable development and research on pharmaceutical care practices. It is also important as a way to communicate to other healthcare professionals effectively. ${ }^{14}$

Development of the harmonised DRP classification system. In February 2019, a workgroup consisting the following 7 Singapore healthcare institutions was convened: Tan Tock Seng Hospital, Khoo Teck Puat Hospital, Institute of Mental Health, National Skin Centre, NHG Pharmacy, National University Hospital and Ng Teng Fong General Hospital. DRP definitions that each institution adopted were obtained for reference to develop a harmonised classification system. The system aims to capture data on identification, causes and resolution of DRP across settings, including inpatient and outpatient acute care hospitals, community hospitals, polyclinics, nursing homes and home-based care. We took a broader definition of DRPs involving medication errors from prescribing and drug administration, as well as operational issues, such as missing instructions or signature in prescriptions, and expired medications.

Published DRP classification systems were reviewed. A widely used classification system recommends that DRPs be broadly categorised as "indication", "adherence", "safety" and "effectiveness" (Table 1). ${ }^{15}$ These systems are multi-focused, and usually have a "problem" and "intervention" sections. ${ }^{16}$ Many of these

\footnotetext{
${ }^{1}$ Division of Pharmacy, Tan Tock Seng Hospital, Singapore

${ }^{2}$ Department of Pharmacy, National University Hospital, Singapore

${ }^{3}$ Department of Pharmacy, Ng Teng Fong General Hospital, Singapore

${ }^{4}$ Department of Pharmacy, Khoo Teck Puat Hospital, Singapore

${ }^{5}$ Department of Pharmacy, Alexandra Hospital, Singapore

${ }^{6}$ Department of Pharmacy, Woodlands Health Campus, Singapore

${ }^{7}$ NHG Pharmacy, National Healthcare Group, Singapore

${ }^{8}$ Institute of Mental Health, Singapore

${ }^{9}$ Singapore Health Services and Outcomes Research, National Healthcare Group, Singapore

${ }^{10}$ Pharmacy Transformation Office, National Healthcare Group Pharmacy, Singapore

${ }^{11}$ Department of Pharmacy, Faculty of Science, National University Singapore

Correspondence: Dr Tat Ming Ng, Division of Pharmacy, Tan Tock Seng Hospital, 11 Jalan Tan Tock Seng, Singapore 308433.

Email: tat_ming_ng@ttsh.com.sg
} 
Table 1. Tasks taken in identifying and resolving broad categories of a drug-related problem (DRP)

\begin{tabular}{ll}
\hline Broad categories of DRP & \multicolumn{1}{c}{ Tasks } \\
\hline Indication (appropriateness) & $\begin{array}{l}\text { Eliminate unnecessary medications. } \\
\text { Initiate medicines for untreated indication. }\end{array}$ \\
\hline Adherence & $\begin{array}{l}\text { Increase patient's willingness to adhere to medication regimen through different methods, } \\
\text { which include motivational interviewing and shared decision making. }\end{array}$ \\
\hline Safety & $\begin{array}{l}\text { Eliminate toxicities. } \\
\text { Identify and/or pre-empt adverse reactions. }\end{array}$ \\
\hline Effectiveness & $\begin{array}{l}\text { Identify most effective medication in a specific patient. } \\
\text { Increase dosages to effective levels. }\end{array}$ \\
\hline
\end{tabular}

systems are modified based on an existing classification system or newly developed. ${ }^{13}$ The DOCUMENT system ${ }^{1}$ comprehensively classifies a DRP and interventions into the type of DRP, action undertaken to investigate it, recommendations made to resolve it, outcome of the actions undertaken and perceived clinical significance of the DRP. We also reviewed the Pharmaceutical Care Network Europe (PCNE) classification system, which is structured similar to the DOCUMENT system. However, these systems may not contain the specific descriptors of DRPs which we are tracking. In the PCNE system, if a wrong diluent was used, the closest "causes" category will be "drug form", thus defining and classifying the cause of the DRP as related to the selection of the drug form. If a need for therapeutic drug monitoring was identified to ensure treatment safety, it will be classified as "others" ${ }^{17}$ The workgroup members felt strongly that explicit categories to describe DRPs of interest in Singapore, needs to be developed. Hence, we adapted and modified both systems for the Singapore context to ensure that the new classification system is able to provide data that ensures continuity in the quality and safety surveillance system of each institution.

The new classification was then tested and refined from May to July 2019. The following additional institutions took part in the testing phase: Alexandra Hospital, Woodlands Health Campus, St Luke's Hospital, Yishun Community Hospital and Jurong Community Hospital. The classification system was tested by pharmacists and pharmacy technicians on a total of 864 DRPs, using data from every participating institutions (data unpublished). Feedback on the DRP categories and their definitions were received, and changes were made to the system collectively.

A hierarchical approach to classify DRPs was developed. The system allows classification of the different aspects of a DRP and the actions taken to resolve it. The system consisted of (1) DRP categories, (2) actions taken to address "indication", "adherence", "safety" and "efficacy" (IASE), (3) process-related causes of the DRP, (4) types of medication errors (adapted from the US National Coordinating Council for Medication Error Reporting and Prevention [NCC MERP] classification) and (5) assessment and recommendations (documentation). ${ }^{14}$ Table 2 describes the relationships of the DRP categories, actions taken and process-related causes. The user first selects the DRP category deemed most appropriate. This is followed by the identification of the action taken to address the "indication", "adherence", "safety" and/ or "efficacy" associated with the DRP. After which, the cause of the DRP associated with the action taken can be selected. Each DRP is then subcategorised as either a medication error (4) or a recommendation to optimise drug therapy. This is followed by a clear and concise documentation of the assessment and recommendation using the SBAR format: situation, background, assessment and recommendation. ${ }^{18}$ The descriptors (1) to (4) can be modified to fit the local electronic medical record systems, such as the Epic electronic medical record system (Epic Systems Corp, Verona, US), while keeping the definition as described in this document

DRP categories. To ensure appropriate classification of each DRP category and actions taken to address the DRP, the team elected to follow a thought process. The verbatim transcriptions for each DRP category are as follows:

A. Drug selection: I started, stopped or changed a drug to....

B. Dosage regimen: I changed dosing regimen by...

C. Preparation and administration: I changed route/site/ diluent/container for....

D. Monitoring: I suggested monitoring or tests because of potential...

E. Adherence and education: I engaged patient/client/ caregiver to.... 
Table 2 describes each DRP and the corresponding actions taken to resolve it based on broad DRP categories. DRPs categorised as "operational" describes the role of pharmacists as well as pharmacy technicians in addressing routine operational aspects of medication use, and hence do not require the reporter to document actions taken under IASE.

Actions taken to address DRPs and process-related causes of DRPs. Prior to developing the harmonised categorisation system, DPR definitions in some institutions were based on process-related causes of the DRP. For example, a wrong medical history taken by the prescriber resulting in a higher dose prescribed was classified as "wrong medical history" rather than "dose and regimen". These processes-related causes of DRPs are routinely reported and tracked by quality and improvement committees of these institutions. In contrast, a DRP can also be caused by "risk of treatment continuation outweighs benefits". There can be a multitude of descriptions for these "clinical causes". DRP can also be caused by patient-related causes (e.g. lack of insight into disease, forgetfulness, etc). In the new categorisation, only process-related causes are defined. Hence, the "cause of DRP (processrelated)" category was added following actions taken to address IASE in this hierarchical system.

However, operational DRPs will not be linked to "actions taken" and "process-related causes" as they refer to problems that are related to routine operational aspects of medication use, and are mainly used for estimation of workload or to drive process improvements. As we had to ensure continuity in the quality and safety surveillance system of each institution, these specific categories were placed under operational DRPs (Table 2).

Defining DRPs that are regarded as medication errors, and documenting assessment and recommendations. A DRP is commonly defined as an event or circumstance involving drug treatment that actually or potentially interferes with the optimal outcome of a patient's medical care. It broadly includes events related to errors, adverse effects or adherence issues. ${ }^{1}$ DRPs can be preventable or unpreventable; and can potentially or actually be caused by an error, intentional or unintentional deviation from accepted drug use, or an unpredictable reaction to an appropriate drug. ${ }^{19}$

We adopted the definition that a medication error is defined as any preventable event in the medication use process that may cause or lead to patient harm while the medication is in the control of the healthcare professional, patient, or consumer. ${ }^{20}$ In an attempt to provide focus and definitions to medication errors, the workgroup developed 2 consensus decisions: (1) as the primary focus is to identify medication errors by healthcare professionals, medication errors caused by the patient or consumers will need to be explicitly stated, and (2) DRPs that can be defined as medication errors will be additionally classified.

The types of medication errors were adapted from NCC MERP and defined:

A. Hazards/Risks (events that have capacity to cause error/harm)

B. Near-misses (error occurred but did not reach patient)

C. Actual Error (reached patient but no harm)

D. Actual Error (reached patient and require monitoring/ intervention to confirm no harm)

E. Actual Error (caused temporary patient harm requiring intervention)

F. Actual Error (caused temporary patient harm requiring initial/prolonged hospitalisation)

G. Actual Error (caused permanent patient harm)

Due to varying reporting needs, some of these medication errors, particularly categories $E$ to $G$, may be reported only in the incident reporting systems of the respective institutions.

We adopted a pragmatic approach in the design and testing of the classification system, and acknowledge that inter-rater reliability and external validity could be evaluated for future research. This DRP classification system will be incorporated in the Next Generation Electronic Medical Record system, which will be progressively deployed across the National Healthcare Group and National University Health System. Further work and improvements to this classification may be needed for other public and private healthcare institutions to adopt the new DRP categorisation system. The harmonisation will allow institutions to build a common framework to identify gaps for innovative care and to characterise the value of pharmacy.

Over time, the collection of large quantities of DRP data with harmonised categorisation, together with natural language processing and machine learning, will form the foundation to establish an accurate algorithm and automate the classification of DRPs by analysing the SBAR documentation. With a better understanding of the DRPs across the Singapore healthcare system, decision support systems based on machine learning can be designed to prevent the occurrence of DRPs at the point of prescribing. 


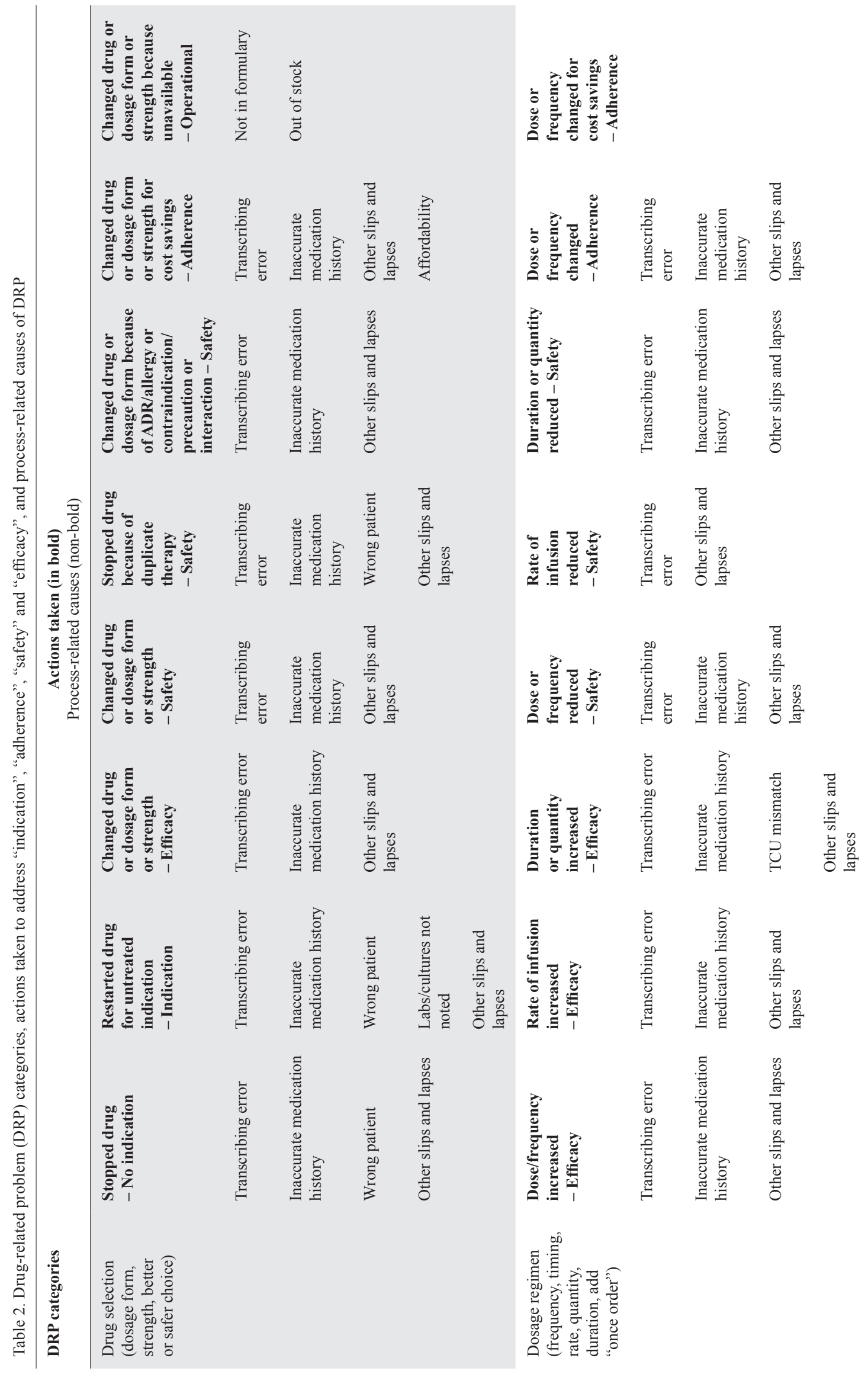




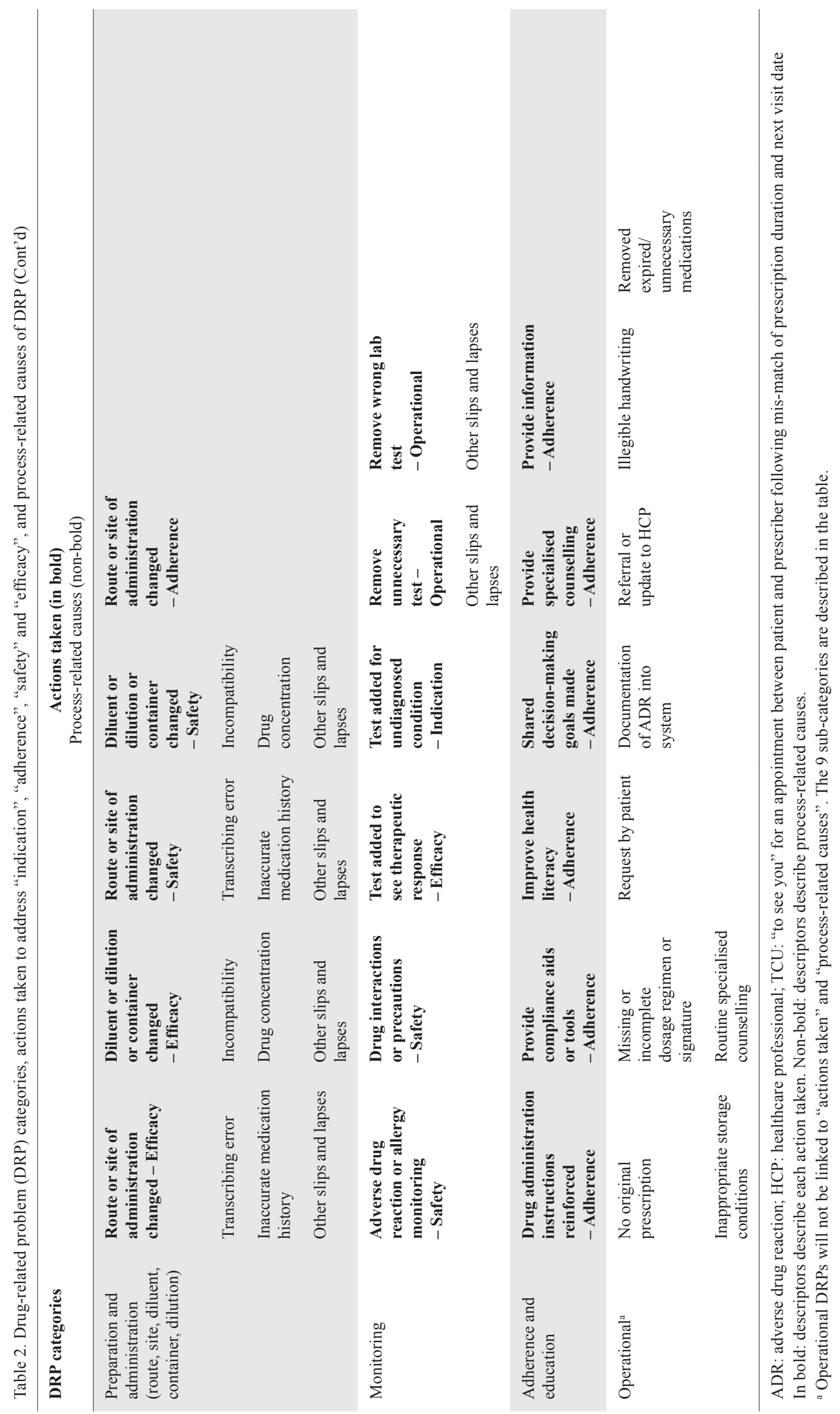




\section{REFERENCES}

1. Williams M, Peterson GM, Tenni PC, et al. DOCUMENT: a system for classifying drug-related problems in community pharmacy. Int J Clin Pharm 2012;34:43-52.

2. Naples JG, Hanlon JT, Schmader KE, et al. Recent literature on medication errors and adverse drug events in older adults. J Am Geriatr Soc 2016;64:401-8.

3. Salvi F, Marchetti A, D'Angelo F, et al. Adverse drug events as a cause of hospitalization in older adults. Drug Saf 2012;35:29-45.

4. López MP, Saliente MT, Company ES, et al. Drug-related problems at discharge: results on the Spanish pharmacy discharge programme CONSULTENOS. Int J Pharm Pract 2010;18:297-304

5. Toh MR, Teo V, Kwan YH, et al. Association between number of doses per day, number of medications and patient's noncompliance, and frequency of readmissions in a multi-ethnic Asian population. Prev Med Rep 2014;1:43-7.

6. Chia HS, Ho JA, Lim BD. Pharmacist review and its impact on Singapore nursing homes. Singapore Med J 2015;56:493-501.

7. Yeo QQ, Chong JBK, Chung WL, et al. Drug Related Problems Detected During a Brown Bag Review by a Pharmacist-Initiated Octo-Pills Programme in Singapore. J Appl Pharm 2017,9:1-4.

8. Ang F, Pau JE, Koh EW, et al. Drug-related problems associated with community-dwelling older persons living alone in Singapore. Int J Clin Pharm 2019;41:719-27.

9. Cheong ST, Ng TM, Tan KT. Pharmacist-initiated deprescribing in hospitalised elderly: prevalence and acceptance by physicians. Eur J Hosp Pharm 2018;25:e35-9.

10. Ng TM, Teo CJ, Heng ST, et al. Impact of round-the-clock pharmacist inpatient medication chart review on medication errors. J Am Coll Clin Pharm 2020;3:1437-43.

11. Woon J, Choo R, Yeo S, et al. Prevalence and Associated Cost of Hospitalization Arising from Preventable Drug-Related
Problems in Singapore: A Cross-Sectional Study. Sch J App Med Sci 2019;7:312-22.

12. Hawes EM, Maxwell WD, White SF, et al. Impact of an outpatient pharmacist intervention on medication discrepancies and health care resource utilization in posthospitalization care transitions. J Prim Care Community Health 2014;5:14-8.

13. Basger BJ, Moles RJ, Chen TF. Application of drug-related problem (DRP) classification systems: a review of the literature. Eur J Clin Pharmacol 2014;70:799-815.

14. Basger BJ, Moles RJ, Chen TF. Development of an aggregated system for classifying causes of drug-related problems. Ann Pharmacother 2015;49:405-18.

15. Patient-Centered Primary Care Collaborative. The PatientCentered Medical Home: Integrating Comprehensive Medication Management to Optimize Patient Outcomes resource guide, Second Edition, June 2012. PCPCC: Washington, DC.

16. van Mil JW, Westerlund LO, Hersberger KE, et al. Drug-related problem classification systems. Ann Pharmacother 2004;38:859-67.

17. Pharmaceutical Care Network Europe Association. Classification for Drug Related Problems V9.1. Available at: https://www.pcne.org/ upload/files/417_PCNE_classification_V9-1_final.pdf. Accessed on 18 April 2021

18. De Meester K, Verspuy M, Monsieurs KG, et al. SBAR improves nurse-physician communication and reduces unexpected death: a pre and post intervention study. Resuscitation 2013;84:1192-6.

19. van Mil JWF, Westerlund T, Brown L, et al. Medical care and drug-related problems: Do doctors and pharmacists speak the same language? Int J Clin Pharm 2016;38:191-4.

20. Hartwig SC, Denger SD, Schneider PJ. Severity-indexed, incident report-based medication error-reporting program. Am J Hosp Pharm 1991;48:2611-6. 